\title{
Electronic combination lock system simulation research
}

\author{
Zhao You Gui \\ Chongqing college of engineering \\ Department of electronic and information engineering, Chongqing 400056 ,China \\ lichengyong000@126.com
}

\begin{abstract}
Keywords: Electronic combination lock system; The simulation; Proteus;Single chip microcomputer
\end{abstract}

\begin{abstract}
In order to achieve the display effect of electronic combination lock system, this paper proposes a Proteus simulation test system design scheme based on single chip microcomputer, and completes the hardware and software design of the system. The hardware part mainly USES the Proteus simulation, virtual instrument software part adopts singlechip c language programming, able to complete visualization simulation of the whole system. Practical application shows that the simulation circuit has the characteristics of simple operation, accurate test, meet the requirements of the system design.
\end{abstract}

\section{Introduction}

In the field of security technology to guard against, with security alarm function of electronic password control system gradually replace the traditional mechanical password control system, overcome the password of mechanical password control quantity is little, the shortcomings of poor safety performance, both in technology and the electronic password control system on the performance are greatly improved. With the development of large scale integrated circuit technology, especially the single chip microcomputer, the advent of the intelligent password control system with microprocessor, it besides has the function of traditional electronic password control system, also introduced the intelligent management, expert analysis system, and other functions, so that the password control system has high security, reliability, is increasingly widely used in .

\section{System design and analysis}

The specific requirements of the electronic combination lock is as follows:

51 series microcontroller P1 pins S1 - the S8, external detached buttons respectively represent Numbers 0 to 5, and determine the keys, the cancel button. SCM from P3.0 - four P3.3 output signal, respectively for a electromagnetic lock drive signal and password error indication, alarm output, has the lock instruction signal, respectively use leds L1 - L4 instructions. P3.4 after active buzzer, used to implement the prompt.

Basic requirements:

(1) the initial password is 123450 , after losing a specified key lock, the cancel button to remove all the input, each key has a short "drop" sound prompt.

Right after (2) the password input, output a electromagnetic lock unlock signals and has the lock, and two short "drop" sound prompt. 4 seconds after the lock signal and reset the lock instruct.

(3) the password input error, sent out a loud "drop" sound, misdirection prompt and password error indicator, three times the password mistake, sirens sound alarm, and password error indicator, alarm indicator, after 15 seconds to enter the password again, after 15 seconds, remove all warning and instructions.

(4) after 5 seconds without any operation, remove all input, waiting for the next input.

Design requirements:

(1) design reasonable, correct;

(2) the system hardware design and welding production;

(3) the system software design and debugging; 
(4) the system alignment.

The main design conditions:

(1) the MCS - 51 SCM experiment work station 1;

(2) the PC and single chip debug software;

(3) 1 set of microcomputer application system board;

(4) to make 1 set of tools;

(5) required for design of the system components.

\section{the overall design}

The system structure diagram as shown in figure 1, the controller with 51 series microcontroller, through matrix key module as input ports, the dynamic digital tube, at the same time control diode prompt functions.

\section{Proteus simulation circuit diagram}

Diversified simulation circuit, the system adopts dynamic digital tube display mode and matrix button control mode, through the bus wiring connected to the node way, is the overall look beautiful and easy, the effect is more obvious, and give the feeling on the vision more realistic, without the actual device. On the effect of algorithm to achieve consistent with the actual, the test on time.

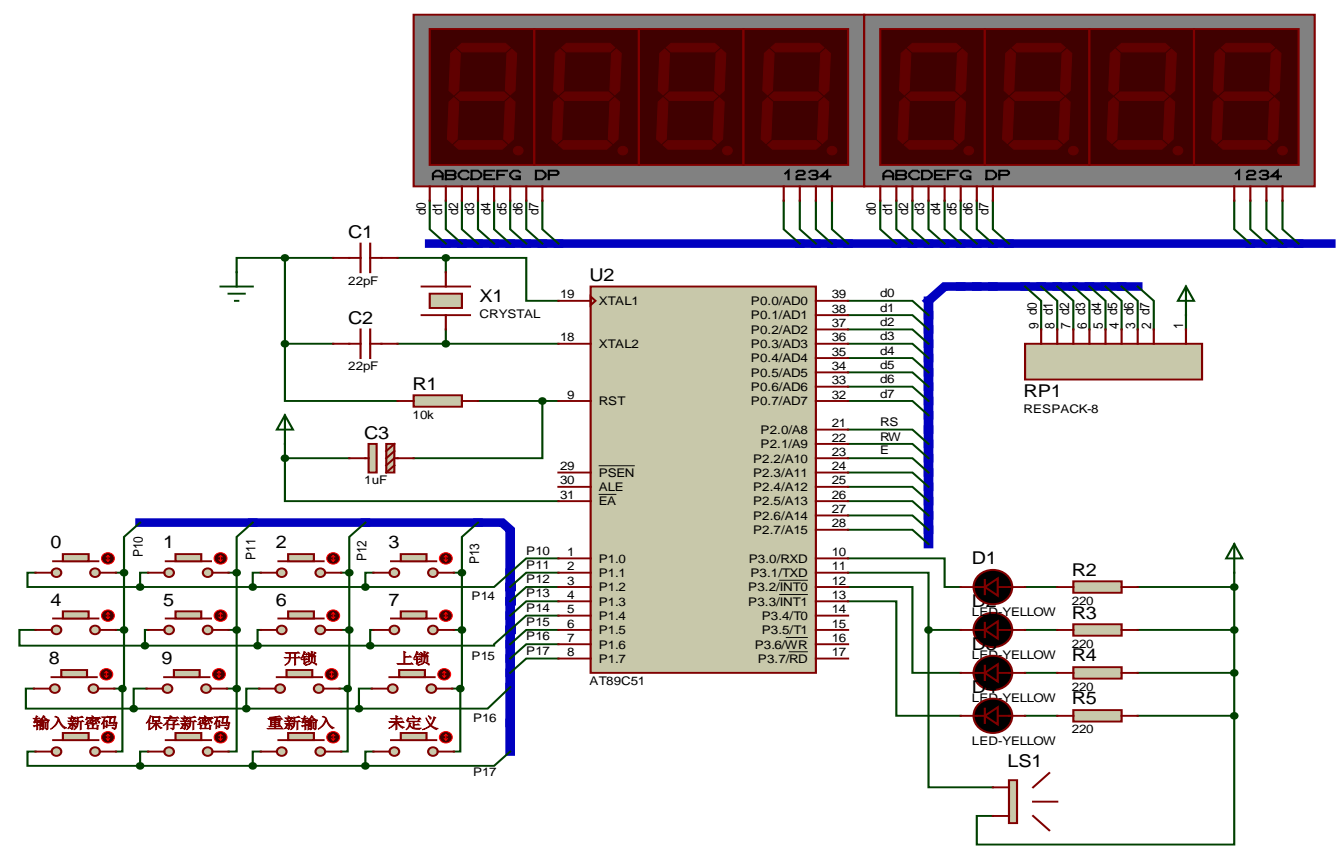

Figure 2 system simulation diagram

Direct load simulation system algorithm design code generated HEX file, with the actual running effect is consistent, without redundant operation, convenient beginners applications.

\section{The test system software design}

The test system software adopts $\mathrm{C}$ programming in a high-level language, programming platform is keil, keil platform is the common software development platform for free. The platform not only provides support for $\mathrm{C}$ language and assembly language, but also has all kinds of test, the ability of communication, control, and numerical analysis, have control, powerful function library function rich, strong real-time performance, easy in programming. In order to update, maintain and expand in the future, in the design process by adopting the idea of modularization, the whole system by 
matrix key module, display module, diode module, timer interrupt module and initialization module, etc.

The overall program design ideas:

Program points as the main program and interrupt service routines to two main parts, the main program to complete the variables and the single chip microcomputer special function register initialization, into a loop structure. In a loop, first of all determine whether button press, if you have any buttons determine whether numeric keys, or function keys, depending on the buttons to perform the corresponding function. Then according to the password is correct judgment situation, carry out the corresponding operation. Loop eventually will need to display content by dynamic scanning on the digital tube display.

Interrupt service routine as long as the realization of the three state of timing, the need when standby time for 5 seconds, password needs to correct timing $5 \mathrm{~s}$, entered an incorrect password three times need timing for 15 seconds. What is the current time, determined by the main program according to the results of password judgment.

Interrupt service routine mainly completed three time the timing of work, including (1) after the button to start the standby time, when the standby for more than $5 \mathrm{~s}$ input content is clear. (2) after the password input the correct timing, remove the lock after $4 \mathrm{~s}$ driving signal and indicating signal has been unlocked. Three (3) the password input error of timing, timing $15 \mathrm{~s}$, in is unable to enter the password again within 15 s, 15 seconds after clear all warning and instructions.

\section{The experimental application}

(1) software debugging scheme

Weifu software, in the new file "file \", the new C language source program files, write the corresponding program. In menu \"file" in new projects, new projects and the C language source program file included in the project file. In the project $\backslash$ compilation menu compiled C source file, check the grammar errors and logic errors. After the successful compilation, produce to the target file "*. Hex" suffix.

(2) the hardware debugging scheme

In the design platform, the MCU P1.0 - P1.7 respectively with eight independent type keyboard through the plug wire connected, will P3.0 - P3.3 respectively with 4 leds connected, P3.4 input connected with a buzzer. In wei don't compile the program file into the target file, will download line installed on the experiment platform of download line interface, run "MCU download program", select the flash data file, click on the "programming" button, download the program files to the MCU flash. Then, electricity to restart the single-chip microcomputer, check whether written procedures to achieve the requirement of the topic, is a comprehensive fully complete the content of the case.

\section{Conclusion}

The test system adopts the common device for hardware platform, software design of the idea of modular design, improve the reliability and maintainability of the system. The test system has been used in the entrance guard system test, the actual application shows that this test system has the test accurate, stable and reliable, friendly man-machine interface and other characteristics, has reached the design requirements.

\section{Acknowledgement}

In this paper, the research was sponsored by the Chongqing city board of education science and technology research projects, based on the weak light signal acquisition detection multi-touch screen display technology research and application (Project No. KJ1401701) Chongqing college of engineering for 13 years campus research projects(Project No. 2013xzky05). 


\section{References}

[1] LIAN Chun-yuan,XU Ai. A source tracking system based onMSP430 MCU[J]. Science \& Technology Association Forum,2010(11):68 - 69.

[2] YU Wei-wei,YAN De-tian,YANG Yue.Research on an automatictracking filter based on MAX260 and its application[J]. ModernElectronics Technique,2008 (5):76 - 78.

[3] ZHAO Xiao-ling.Accomplishment of fingerprint safe software based on MSP430 microcontroller[J].SAMSON,2007,(11):38.

[4] SUI Bi-xia. Dynamic Scanning LED Display with the Serial Portof Single chip[J].Electroic Engineer,2006,32(6):31-32.

[5] XIA Jun,WANG Tian.Remote control timer switch based on STM32and GSM[J]. Electronic Science and Technology,2013(1):112-114,130.

[6] Zhang, Hongwei,Wang, Xin-Huan,Wang, Fu-Zhong."Research on microcomputer serial courses diversified teaching mode,". 20102 nd International Conference on Education Technology and Computer,ICETC . 2010

[7] Wang, Xinhuan,Zhang, Hongwei,Gao, Qinghua,Zhang, Wei."The construction of single-chip microcomputer virtual experiment platform based on proteus,". ICCSE 2010 - 5th International Conference on Computer Science and Education, Final Program and Book of Abstracts . 2010

[8] Zheng Yamin."The reform and practice on the course of principle and interfaces technology of microcomputer,". Proceedings of the 1 st International Workshop on Education Technology and Computer Science, ETCS . 2009 